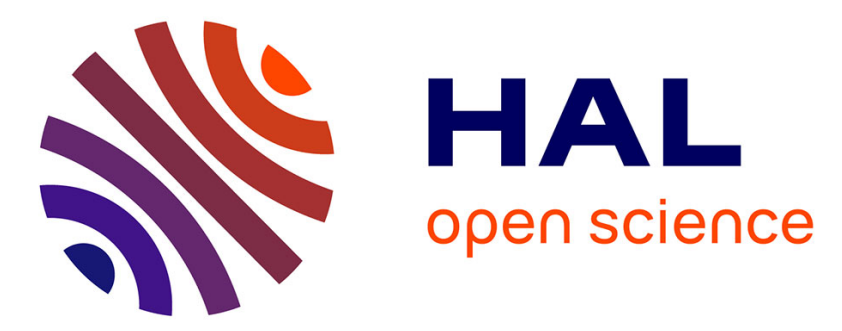

\title{
Competition among non-life insurers under solvency constraints: A game-theoretic approach
}

Christophe Dutang, Hansjoerg Albrecher, Stéphane Loisel

\section{To cite this version:}

Christophe Dutang, Hansjoerg Albrecher, Stéphane Loisel. Competition among non-life insurers under solvency constraints: A game-theoretic approach. European Journal of Operational Research, 2013, 231 (3), pp.702 - 711. 10.1016/j.ejor.2013.06.029 . hal-01616156

\section{HAL Id: hal-01616156 \\ https://hal.science/hal-01616156}

Submitted on 13 Oct 2017

HAL is a multi-disciplinary open access archive for the deposit and dissemination of scientific research documents, whether they are published or not. The documents may come from teaching and research institutions in France or abroad, or from public or private research centers.
L'archive ouverte pluridisciplinaire HAL, est destinée au dépôt et à la diffusion de documents scientifiques de niveau recherche, publiés ou non, émanant des établissements d'enseignement et de recherche français ou étrangers, des laboratoires publics ou privés. 


\title{
Competition among non-life insurers under solvency constraints: a game-theoretic approach
}

\author{
C. Dutang*† H. Albrecher $\stackrel{\ddagger}{\ddagger}$ S. Loisel*
}

\begin{abstract}
We formulate a noncooperative game to model competition for policyholders among non-life insurance companies, taking into account market premium, solvency level, market share and underwriting results. We study Nash equilibria and Stackelberg equilibria for the premium levels, and give numerical illustrations.
\end{abstract}

Keywords: Non-life insurance; Market model; Game theory; Nash equilibrium; Stackelberg equilibrium

\section{Introduction}

Insurance pricing is a classical topic for both actuaries and academics. Standard actuarial approaches for non-life insurance typically suggest to use expectation, standard deviation, or quantiles of the underlying risk to determine a suitable premium. For an overview of principles of premium calculation, see e.g. Teugels \& Sundt (2004). The resulting (socalled technical) premium is then often altered by marketing and management departments, and actual deviations from the technical premium can be considerable. Affordability by customers and mutualization across the portfolio are often used as arguments to justify that policyholders do not necessarily pay the risk-based premium. But another important reason for such deviations from the technical premium is the dependency on market conditions. In order to study that factor, a market model is needed to study the economic interactions between insurers and policyholders.

Basic economic models suggest that the equilibrium premium is the marginal cost, as any upward deviation from this premium equilibrium will result in losing all the policies

\footnotetext{
${ }^{*}$ Université de Lyon, Université Claude Bernard Lyon 1, Institut de Science Financière et d'Assurances, 50 Avenue Tony Garnier, F-69007 Lyon, France

${ }^{\dagger}$ Institut de Recherche Mathématique Avancée, UMR 7501, Université de Strasbourg et CNRS, 7 rue René Descartes, F-67000 Strasbourg, France

${ }^{\ddagger}$ Department of Actuarial Science, Faculty of Business and Economics, University of Lausanne, UNILDorigny, 1015 Lausanne, Switzerland.
} 
in the next period. Other advanced economic models generally focus on moral hazard and adverse selection. The Rothschild and Stiglitz's model (see Rothschild \& Stiglitz (1976)) deals with a utility-based agent framework where policyholders have private information on their own risk. In this model, insurers provide a menu of contracts, i.e. pairs of premium and deductible, from which policyholder can freely choose. At the equilibrium, individuals with low risk aversion choose full coverage, whereas individuals with high risk aversion are more attracted to partial coverage. Note that an equilibrium price may not exist if all insurers offer just one type of contract. Picard (2009) considers an extension by allowing insurers to offer participating contracts (such as mutual-type contracts). This feature guarantees the existence of an equilibrium, which reveals the risk level of the policyholders. An important area of applications for such models is health insurance, where moral hazard and adverse selection play a major role, see e.g. Geoffard et al. (1998), Wambach (2000) and Mimra \& Wambach (2010). But, in practice customers do not move from one insurer to a cheaper one as swiftly as economic models anticipate. The inertia of the insurance demand prevents policyholders to always look for the cheapest insurer, see Smith et al. (2000) for a case study in Australia. Accordingly, the customer behavior is much more complicated.

Moreover, the economic models mentioned above are not able to incorporate some insurance market features. Taylor $(1986,1987)$ deals with underwriting strategies of insurers and provides first attempts to model optimal responses of an insurer to the market on a given time horizon, see also Kliger \& Levikson (1998), Emms et al. (2007), Moreno-Codina \& Gomez-Alvado (2008) for extensions. All these papers focus on one single insurer and in that way assume that insurers are playing a game against an impersonal market player, so that the market price is independent of their own actions.

In this paper, we want to further investigate the suitability of game theory for insurance market modelling. The use of game theory in actuarial science has a long history dating back to K. Borch and J. Lemaire, who mainly used cooperative games to model risk transfer between insurer and reinsurer, see e.g. Section 3.1 of Brockett \& Xia (1995) for a review. Among earlier work using noncooperative game theory to model the non-life insurance market, two kinds of models were pursued: the Bertrand oligopoly where insurers set premiums and the Cournot oligopoly where insurers choose optimal values of insurance coverage. Polborn (1998) considers a Bertrand model in which rational consumers maximize their utility function and for which the equilibrium premium is the expected loss. Rees et al. (1999) extend this model by requiring insurers to announce both a premium and a capital value. Under rational behavior, the premium equilibrium remains the expected loss and the capital equilibrium avoids insolvency. Powers \& Shubik (1998) propose a Cournot model with two types of players. Policyholders state the amount that they are willing to pay, and insurers state the amount of risk they are willing to underwrite. Based on a clearing-house system to determine the market price, each player maximizes its expected utility. Assuming risk neutral insurers and risk averse consumers, the resulting premium equilibrium is larger than the expected loss. They also study scale effects of the number of insurers on the premium equilibrium. Powers \& Shubik (2006) include reinsurers as additional players and study the optimal number of reinsurers in an insurance market. 
The present paper aims to model competition in non-life insurance markets with noncooperative game theory in order to extend the insurer-vs-market reasoning of Taylor (1986, 1987). We extend the Bertrand model of Rees et al. (1999) by considering a lapse model and an aggregate loss model for policyholders. The lapse model describes the policyholder behavior through a lapse probability which is a function of the premiums offered by the insurers. We also consider a solvency constraint function for insurers. As a main result, we show that incorporating competition when setting premiums leads to a significant deviation of Nash and Stackelberg equilibria both from the actuarial premium and a one-insurer optimized premium.

The rest of the paper is organized as follows. Section 2 develops the one-period noncooperative game of this paper. Existence and uniqueness of a premium equilibrium are established. Section 3 presents numerical illustrations of the game. A conclusion and perspectives are given in Section 4.

\section{A one-period model}

Consider $I$ insurers competing in a market of $n$ policyholders with one-year contracts $(n$ is fixed). The policyholders are assumed to react to price changes (either stay with the present insurer or switch to one of the competitors), but do not have any other influence on the premium level (which is a realistic assumption, in particular for personal lines of business such as compulsory third-party motor liability). In view of the one-year time horizon and the randomness of claim sizes, this model focuses on non-life insurance products (i.e. products for which the claim event is not linked to the life of the policyholder).

The "game" for insurers is to set the premium for which policies are offered to the policyholders. Let $\left(x_{1}, \ldots, x_{I}\right) \in \mathbb{R}^{I}$ be a price vector, with $x_{j}$ representing the premium of Insurer $j$. Once the premium is set by all insurers, the policyholders choose to renew or to lapse from their current insurer. Then, insurers pay occuring claims during the coverage year. At the end of the period, underwriting results are determined, and the insurer capital is updated: some insurers may be bankrupt. As we deal with a one-period model, for simplicity we do not consider investment results.

In the next subsections, we present the four components of the game: (i) a lapse model, (ii) a loss model, (iii) an objective function and (iv) a solvency constraint function. These four components are critical factors for the analysis of the non-life insurance market, see e.g. IASB (2008). In the sequel, a subscript $j \in\{1, \ldots, I\}$ will always denote an insurer index, whereas a subscript $i \in\{1, \ldots, n\}$ denotes policyholder index. In the sequel, "insurer" is used when referring to players of the insurance game. 


\section{$2.1 \quad$ Lapse model}

In this subsection, we present our lapse model which is designed as a compromise between reflecting the policyholders' behavior in a reasonable way, yet keeping mathematical tractability. Let $n_{j}$ be the initial portfolio size of Insurer $j$ ( $\operatorname{such}$ that $\sum_{j=1}^{I} n_{j}=n$ ). It seems natural that the choice of policyholders for an insurer is highly influenced by the choice of the previous period. We assume that the dispatch (among the $I$ insurers) of the $n_{j}$ policyholders of Insurer $j$ follows an $I$-dimensional multinomial distribution $\mathcal{M}_{I}\left(n_{j}, p_{j \rightarrow}(x)\right)$ with probability vector $p_{j \rightarrow}(x)=\left(p_{j \rightarrow 1}(x), \ldots, p_{j \rightarrow I}(x)\right)$. The probability $p_{j \rightarrow k}(x)$ to move from Insurer $j$ to Insurer $k$ naturally depends on the price vector $x$, (concretely, the difference of premiums). Empirically, the probability to lapse $p_{j \rightarrow k}(x)($ with $k \neq j)$ is generally much lower than the probability to renew $p_{j \rightarrow j}(x)$. To our knowledge, only the UK market shows lapse rates above $50 \%$, cf. Dreyer (2000).

In the economics literature, $p_{j \rightarrow k}$ is considered in the framework of discrete choice models. In the random utility maximization setting, McFadden (1981) or Anderson et al. (1989) propose multinomial logit and probit probability choice models. In this paper, we choose a multinomial logit model, because of its simplicity (the probit link function, based on the multivariate normal distribution, would not significantly change the shape of the lapse function). Working with unordered choices, we arbitrarily set the insurer reference category for $p_{j \rightarrow k}$ to $j$, the current insurer. We define the probability for a customer to go from insurer $j$ to $k$ given the price vector $x$ by the multinomial logit model

$$
p_{j \rightarrow k}(x)= \begin{cases}\frac{1}{1+\sum_{l \neq j} e^{f_{j}\left(x_{j}, x_{l}\right)}} & \text { if } j=k, \\ \frac{e^{f_{j}\left(x_{j}, x_{k}\right)}}{1+\sum_{l \neq j} e^{f_{j}\left(x_{j}, x_{l}\right)}} & \text { if } j \neq k,\end{cases}
$$

where the sum is taken over the set of insurers $\{1, \ldots, I\}$ and $f_{j}$ is a price-sensitivity function. We consider two types of price functions

$$
\bar{f}_{j}\left(x_{j}, x_{l}\right)=\bar{\mu}_{j}+\bar{\alpha}_{j} \frac{x_{j}}{x_{l}} \text { and } \tilde{f}_{j}\left(x_{j}, x_{l}\right)=\tilde{\mu}_{j}+\tilde{\alpha}_{j}\left(x_{j}-x_{l}\right) .
$$

The first function $\bar{f}_{j}$ assumes a price-sensitivity according to the ratio of proposed premium $x_{j}$ and competitor premium $x_{l}$, whereas $\tilde{f}_{j}$ works with the premium difference $x_{j}-x_{l}$. Parameters $\mu_{j}, \alpha_{j}$ represent a base lapse level and price-sensitivity, respectively. We assume that insurance products display positive price elasiticity of demand $\alpha_{j}>0$. One can check that $\sum_{k} p_{j \rightarrow k}(x)=1$.

Equation (1) can be rewritten as

$$
p_{j \rightarrow k}(x)=p_{j \rightarrow j}(x)\left(\delta_{j k}+\left(1-\delta_{j k}\right) e^{f_{j}\left(x_{j}, x_{k}\right)}\right),
$$

with $\delta_{i j}$ denoting the Kronecker delta. It is difficult to derive general properties of the distribution of a sum of binomial variables with different probability parameters, except when the size parameters $n_{j}$ are reasonably large, in which case the normal approximation is appropriate. 
The portfolio size $N_{j}(x)$ of insurer $j$ for the next period is a random variable determined by the sum of renewed policies and (new) policyholders coming from other insurers. Hence, $N_{j}(x)$ is a sum of $I$ independent binomial variables $\left(B_{k j}\right)_{k}$ with parameters $n_{k}, p_{k \rightarrow j}(x)$

$$
N_{j}(x)=B_{j j}(x)+\sum_{k=1, k \neq j}^{I} B_{k j}(x) .
$$

This assumption is in contrast to the standard models in classical ruin theory, where the portfolio size is assumed constant over time (see e.g. Asmussen \& Albrecher (2010) for a recent survey and Malinovskii (2010) for an attempt to have a premium-dependent portfolio size). With this insurer choice model, the expected portfolio size of insurer $j$ reduces to

$$
\mathbb{E}\left(N_{j}(x)\right)=n_{j} \times p_{j \rightarrow j}(x)+\sum_{l \neq j} n_{l} \times p_{j \rightarrow k}(x) .
$$

\subsection{Loss model}

Let $Y_{i}$ be the aggregate loss of policy $i$ during the coverage period. We assume no adverse selection among policyholders of any insurers, i.e. $Y_{i}$ are independent and identically distributed (i.i.d.) random variables, for all $i=1, \ldots, n$. Let us assume a simple frequency average severity loss model

$$
Y_{i}=\sum_{l=1}^{M_{i}} Z_{i, l}
$$

where the claim number $M_{i}$ is Policyholder $i$. The claim severities $\left(Z_{i, l}\right)_{l}$ are i.i.d. as some generic random variable $Z$. The aggregate claim amount for Insurer $j$ is then

$$
S_{j}(x)=\sum_{i=1}^{N_{j}(x)} Y_{i}=\sum_{i=1}^{N_{j}(x)} \sum_{l=1}^{M_{i}} Z_{i, l},
$$

where $N_{j}(x)$ is the portfolio size defined in Equation (3). We consider two claim number distributions: (i) $M_{i}$ follows a Poisson distribution $\mathcal{P}(\lambda)$ and (ii) $M_{i}$ follows a negative binomial distribution $\mathcal{N B}(r, p)$. These instances of the frequency - average severity model are such that the aggregate claim amount $S_{j}(x)=\sum_{i=1}^{N_{j}(x)} Y_{i}$ is still a compound distribution of the same kind, since $Y_{i}$ are assumed i.i.d. random variables. Hence, the aggregate claim amount $S_{j}(x)$ of Insurer $j$ is a compound distribution

$$
S_{j}(x)=\sum_{l=1}^{\widetilde{M}_{j}(x)} Z_{l}
$$

where all claim severities $Z_{l} \stackrel{\text { i.i.d. }}{\sim} Z$ and the claim number $\widetilde{M}_{j}(x)$ follows either a Poisson distribution $\mathcal{P}\left(N_{j}(x) \lambda\right)$ or a negative-binomial distribution $\mathcal{N} \mathcal{B}\left(N_{j}(x) r, p\right)$. In the numerical applications, these two loss models are denoted PLN and NBLN, respectively. For the 
distribution of $Z$, we choose the lognormal distribution, which is due to its heavy-tailedness and at the same time pleasant statistical properties is a popular modeling assumption in many lines of non-life insurance. This choice is by no means crucial, as any other can be handled in an analogous way.

\subsection{Objective function}

In the two previous subsections, we presented two components of the insurance markets: the lapse model (how policyholders react to premium changes) and the loss model (how policyholders face claims). We now turn our attention to the underwriting strategy of insurers, i.e. on how they set premiums.

In Section 2.1, we assumed that price elasticity of demand for the insurance product is positive. Thus, if the whole market underwrites at a loss, any actions of a particular insurer to get back to profitability will result in a reduction of his business volume. This has two consequences for the choice of the objective function: (i) it should involve a decreasing demand function of price $x_{j}$ given the competitors price vector $x_{-j}=\left(x_{1}, \ldots, x_{j-1}, x_{j+1}, \ldots, x_{I}\right)$ and (ii) it should depend on an assessment of the insurer break-even premium $\pi_{j}$ per unit of exposure.

The parameter $\pi_{j}$ corresponds to the estimated mean but depends on the assessment of loss expectation by insurer $j$. We thus define $\pi_{j}$ as

$$
\pi_{j}=\omega_{j} \bar{a}_{j, 0}+\left(1-\omega_{j}\right) \bar{m}_{0}
$$

where $\bar{a}_{j, 0}$ is the actuarial premium based on the past loss experience of insurer $j, \bar{m}_{0}$ is the market premium, available for instance, via rating bureaus or through insurer associations and $\omega_{j} \in[0,1]$ is the credibility factor of insurer $j .{ }^{*} \omega_{j}$ reflects the confidence of insurer $j$ in its own loss experience: the closer to 1 , the more confident insurer $j$ is. Note that $\pi_{j}$ takes into account expenses implicitly via the actuarial and the market premiums.

We choose the demand function as

$$
D_{j}(x)=\frac{n_{j}}{n}\left(1-\beta_{j}\left(\frac{x_{j}}{m_{j}(x)}-1\right)\right),
$$

where $\beta_{j}>0$ is the elasticity parameter and $m_{j}(x)$ is a market premium proxy. The demand $D_{j}(x)$ is not restricted to $\left[0, n_{j} / n\right]$, and thus $D_{j}$ targets both renewal and new business. In this form, $D_{j}(x)$ approximates the expected market share $\mathbb{E}\left(N_{j}(x)\right) / n$ presented in Section 2.1. As the elasticity parameter $\beta_{j}$ is positive, a premium increase (of insurer $j$ ) will result in a decrease of the demand for insurance. The market proxy used in Equation (4) is the

\footnotetext{
${ }^{*}$ Rating bureaus or rating agencies are organizations collecting statistical data from insurers in order to publish market information both for insurers and policyholders. The credibility factor is the weight given to individual loss experience in contrast to collective loss data.
} 
mean price of the other competitors

$$
m_{j}(x)=\frac{1}{I-1} \sum_{k \neq j} x_{k}
$$

The market proxy aims to assess other insurer premiums without specifically targeting one competitor. It can be interpreted as the premium of an ideal medium competitor. Consequently, Insurer $j$ typically does not target the cheapest, the most expensive or the leader insurers.

Now we can state our objective function. We suppose that Insurer $j$ maximizes the expected profit of next year policies which we here define in the multiplicative form

$$
O_{j}(x)=\frac{n_{j}}{n}\left(1-\beta_{j}\left(\frac{x_{j}}{m_{j}(x)}-1\right)\right)\left(x_{j}-\pi_{j}\right),
$$

i.e. the product of the demand $D_{j}$ and the expected profit per policy, representing a companywide expected profit. Thus, maximising the objective function $O_{j}$ leads to a trade-off between increasing premium to favour higher projected profit margins and decreasing premium to defend the current market share. Note that $O_{j}$ has the nice property to be infinitely differentiable with respect to $x$.

\subsection{Solvency constraint function}

Another key feature of the model is a solvency constraint the goal of which is to require insurers to hold a certain amount of capital in order to protect policyholders against adverse collective claim experience. Therefore, in addition to maximizing a certain objective function, insurers must satisfy a solvency constraint imposed by the regulator. A reasonable criterion to find the minimum capital requirement is linked to deviations of the aggregate losses from its expected value, concretely the difference of a high-level quantile and the mean of the loss distribution. For simplicity, this quantity is supposed to be a linear function of the standard deviation of the loss distribution. In practice, the solvency capital is also required on a prospective basis; we take here the simplifying assumption to use only the in-force policy number. Thus, we define the solvency constraint function as

$$
g_{j}^{1}\left(x_{j}\right)=\frac{K_{j}+n_{j}\left(x_{j}-\pi_{j}\right)\left(1-e_{j}\right)}{k \sigma(Y) \sqrt{n_{j}}}-1,
$$

where $k$ is the solvency coefficient chosen to approximate a $99.5 \%$ quantile and $e_{j}$ denotes the expense rate. The numerator corresponds to the sum of the current capital $K_{j}$ and the expected profit on the in-force portfolio, whereas the denominator approximates the required capital. The constraint $g_{j}^{1}(x) \geq 0$ is equivalent to $K_{j}+n_{j}\left(x_{j}-\pi_{j}\right)\left(1-e_{j}\right) \geq k \sigma(Y) \sqrt{n_{j}}$, but $g_{j}^{1}$ is normalized with respect to capital, providing better numerical stability. In $\mathrm{A}$, we give details about the choice of the constant $k$. 
In addition to the solvency constraint, we need to impose bounds on the possible premium. A first choice could be simple linear constraints as $x_{j}-\underline{x} \geq 0$ and $\bar{x}-x_{j} \geq 0$, where $\underline{x}$ and $\bar{x}$ represent the minimum and the maximum premium, respectively. However, the following equivalent reformulation is numerically more stable:

$$
g_{j}^{2}\left(x_{j}\right)=1-e^{-\left(x_{j}-\underline{x}\right)} \geq 0 \text { and } g_{j}^{3}\left(x_{j}\right)=1-e^{-\left(\bar{x}-x_{j}\right)} \geq 0 .
$$

The bounds $\underline{x}$ and $\bar{x}$ could for instance be justified by a prudent point of view of a government regulator. In the sequel, we set $\underline{x}=\mathbb{E}(Y) /\left(1-e_{\text {min }}\right)<\bar{x}=3 \mathbb{E}(Y)$, where $e_{\min }$ is the minimum expense rate.

Summarizing, the constraint function $g_{j}\left(x_{j}\right)=\left(g_{j}^{l}\left(x_{j}\right)\right)_{1 \leq l \leq 3}$ for Insurer $j$ is

$$
\left\{x_{j}, g_{j}\left(x_{j}\right) \geq 0\right\}=\left\{x_{j} \in[\underline{x}, \bar{x}], K_{j}+n_{j}\left(x_{j}-\pi_{j}\right)\left(1-e_{j}\right) \geq k_{995} \sigma(Y) \sqrt{n_{j}}\right\} .
$$

\subsection{Solution concepts}

We consider two solution concepts for our game: the Nash equilibrium for which it is assumed that insurer actions are taken simultaneously, and the Stackelberg equilibrium for which actions take place sequentially with a leader, see e.g. Fudenberg \& Tirole (1991), Osborne \& Rubinstein (2006).

Definition (Nash equilibrium). For a game with $I$ insurers, with payoff functions $O_{j}$ and action set $X_{j}$, a Nash equilibrium is a vector $x^{\star}=\left(x_{1}^{\star}, \ldots, x_{I}^{\star}\right)$ such that for all $j=1, \ldots, I$, $x_{j}^{\star}$ solves the subproblem

$$
\sup _{x_{j} \in X_{j}} O_{j}\left(x_{j}, x_{-j}^{\star}\right)
$$

where $x_{j}$ and $x_{-j}$ denote the action of insurer $j$ and the other insurers' actions, respectively. The action set $X_{j}$ of Insurer $j$ may be parametrized as $X_{j}=\left\{x_{j}, g_{j}\left(x_{j}\right) \geq 0\right\}$.

A Nash equilibrium can hence be interpreted as a point at which no insurer has an incentive to deviate, given the actions of the other insurers. Now, we turn to the definition of a Stackelberg equilibrium. For simplicity, we assume there is one unique leader (Insurer 1) and insurers $j \in\{2, \ldots, I\}$ are followers.

Definition (Stackelberg equilibrium). For a game with one leader and I-1 followers, with payoff functions $O_{j}$ and action set $X_{j}$, a Stackelberg equilibrium is a vector $x^{\star}=\left(x_{1}^{\star}, \ldots, x_{I}^{\star}\right)$ such that $x_{1}^{\star}$ solves the problem

$$
\sup _{x_{1} \in X_{1}} O_{1}\left(x_{1}, x_{-1}^{\star}\left(x_{1}\right)\right)
$$

and $x_{-1}^{\star}\left(x_{1}\right)$ is a Nash equilibrium for the subgame with the $I-1$ followers and given action $x_{1}$ for insurer 1 . 


\subsection{Game sequence}

For our game with objective functions $O_{j}$ in (5) and constraint functions $g_{j}$ in $(7)$, the game sequence is given as follows

(i) Insurers set their premium according to a Nash or a Stackelberg equilibrium $x^{\star}$.

(ii) Policyholders randomly choose their new insurer according to probabilities $p_{k \rightarrow j}\left(x^{\star}\right)$ : we get $N_{j}\left(x^{\star}\right)$.

(iii) For the one-year coverage, claims are random according to the frequency -average severity model relative to the portfolio size $N_{j}\left(x^{\star}\right)$.

(iv) Finally the underwriting result is determined by $U W_{j}\left(x^{\star}\right)=N_{j}\left(x^{\star}\right) x_{j}^{\star}\left(1-e_{j}\right)-S_{j}\left(x^{\star}\right)$ and new capital is $K_{j}+U W_{j}\left(x^{\star}\right)$, where $e_{j}$ denotes the expense rate and $K_{j}$ the initial capital value.

\subsection{Properties of the premium equilibrium}

In this subsection, we investigate properties of the premium equilibrium. We start by showing existence and uniqueness of a Nash equilibrium. Then, we focus on the sensitivity analysis on model parameters of such an equilibrium. Finally, we study the existence of a Stackelberg equilibrium.

Proposition 2.1. The insurance game with I insurers whose objective functions and solvency constraint functions are defined in Equations (5) and (7), respectively, admits a unique Nash premium equilibrium.

Proof. The strategy set is $R=[\underline{x}, \bar{x}]^{I}$, which is nonempty, convex and compact. Given $x_{-j} \in[\underline{x}, \bar{x}]$, the function $x_{j} \mapsto O_{j}(x)$ is a quadratic function with second-degree term $-\beta_{j} x_{j}^{2} / m_{j}(x)<0$ up to a constant $n_{j} / n$. Thus, this function is (strictly) concave. Moreover, for all insurers, the constraint functions $g_{j}^{1}$ are linear functions, hence also concave. By Theorem 1 of Rosen (1965), the game admits a Nash equilibrium, i.e. existence is guaranteed. By Theorem 2 of Rosen (1965), uniqueness is verified if we have the following inequality for all $x, y \in R$,

$$
\sum_{j=1}^{I} r_{j}\left(x_{j}-y_{j}\right) \nabla_{x_{j}} O_{j}(y)+\sum_{j=1}^{I} r_{j}\left(y_{j}-x_{j}\right) \nabla_{x_{j}} O_{j}(x)>0,
$$

for some $r \in \mathbb{R}^{I}$ with strictly positive components $r_{i}>0$. As the function $x_{j} \mapsto O_{j}(x)$ is a strictly concave and differentiable function for all $x_{-j}$, we have $\nabla_{x_{j}} O_{j}(x)\left(y_{j}-x_{j}\right)>$ $O_{j}(y)-O_{j}(x)$ and equivalently $\nabla_{x_{j}} O_{j}(y)\left(x_{j}-y_{j}\right)>O_{j}(x)-O_{j}(y)$. Thus,

$$
\left(x_{j}-y_{j}\right) \nabla_{x_{j}} O_{j}(y)+\left(y_{j}-x_{j}\right) \nabla_{x_{j}} O_{j}(x)>O_{j}(y)-O_{j}(x)+O_{j}(x)-O_{j}(y)=0 .
$$

Taking $r=(1, \ldots, 1)$, Equation (8) is verified. 
Proposition 2.2. Let $x^{\star}$ be the Nash premium equilibrium of the insurance game with I insurers. For each Insurer $j$, the insurer equilibrium $x_{j}^{\star}$ with $\left.x_{j}^{\star} \in\right] \underline{x}, \bar{x}[$ depends on the parameters in the following way: it increases with break-even premium $\pi_{j}$, solvency coefficient $k_{995}$, loss standard deviation $\sigma(Y)$, expense rate $e_{j}$ and decreases with sensitivity parameter $\beta_{j}$ and capital $K_{j}$. When $x_{j}^{\star}=\underline{x}$ or $\bar{x}$, the premium equilibrium is independent of those parameters.

Proof. The premium equilibrium $x_{j}^{\star}$ of insurer $j$ solves the necessary Karush-Kuhn-Tucker conditions:

$$
\begin{aligned}
& \nabla_{x_{j}} O_{j}\left(x^{\star}\right)+\sum_{1 \leq l \leq 3} \lambda_{l}^{j \star} \nabla_{x_{j}} g_{j}^{l}\left(x_{j}^{\star}\right)=0, \\
& 0 \leq \lambda^{j \star}, g_{j}\left(x_{j}^{\star}\right) \geq 0, g_{j}\left(x_{j}^{\star}\right)^{T} \lambda^{j \star}=0,
\end{aligned}
$$

where $\lambda^{j \star} \in \mathbb{R}^{3}$ are Lagrange multipliers, see e.g. Facchinei \& Kanzow (2009). In the last part of equation $(9), g_{j}\left(x_{j}^{\star}\right)^{T} \lambda^{j \star}=0$ is the complementarity equation implying that the $l$ th constraint $g_{j}^{l}$ is either active $\left(g_{j}^{l}\left(x_{j}^{\star}\right)=0\right)$ or inactive $\left(g_{j}^{l}\left(x_{j}^{\star}\right)>0\right)$, but $\lambda_{l}^{j \star}=0$.

We suppose that $\left.x_{j}^{\star} \in\right] \underline{x}, \bar{x}\left[\right.$. Hence, $\lambda_{2}^{j \star}=\lambda_{3}^{j \star}=0$. There are two cases: either the solvency constraint $g_{j}^{1}$ is active or not. Let us assume the solvency constraint is inactive. Insurer $j$ 's premium equilibrium verifies $\nabla_{x_{j}} O_{j}\left(x^{\star}\right)=0$, i.e.

$$
\frac{n_{j}}{n}\left(1-2 \beta_{j} \frac{x_{j}^{\star}}{m_{j}\left(x^{\star}\right)}+\beta_{j}+\beta_{j} \frac{\pi_{j}}{m_{j}\left(x^{\star}\right)}\right)=0 .
$$

Let $x_{y}^{j}$ be the premium vector with the $j$ th component equal to $y$, i.e. $x_{y}^{j}=\left(x_{1}, \ldots, x_{j-1}\right.$, $\left.y, x_{j+1}, \ldots, x_{I}\right)$. Given a parameter $z$ on which we want to investigate the sensitivity, we define the function $F_{x}^{j}$ as

$$
F_{x}^{j}(z, y)=\frac{\partial O_{j}}{\partial x_{j}}\left(x_{y}^{j}, z\right)
$$

where the objective function (also) depends on $z$. Equation (10) can be simply rewritten as $F_{x^{\star}}^{j}\left(z, x_{j}^{\star}\right)=0$.

By the continuous differentiability of $F$ with respect to $z$ and $y$ and the fact that $F_{x}^{j}(z, y)=0$ has at least one solution $\left(z_{0}, y_{0}\right)$, we can invoke the implicit function theorem, see e.g. Zorich (2000). So there exists a function $\varphi$ defined in a neighborhood of $\left(z_{0}, y_{0}\right)$ such that $F_{x}^{j}(z, \varphi(z))=0$ and $\varphi\left(z_{0}\right)=y_{0}$. Furthermore, if $\frac{\partial F_{x}^{j}}{\partial y}\left(z_{0}, y_{0}\right) \neq 0$, the derivative of $\varphi$ is given by

$$
\varphi^{\prime}(z)=-\left.\frac{\frac{\partial F_{x}^{j}}{\partial z}(z, y)}{\frac{\partial F_{x}^{j}}{\partial y}(z, y)}\right|_{y=\varphi(z)} .
$$

In our case, we have

$$
\frac{\partial F_{x}^{j}}{\partial y}(z, y)=\frac{\partial^{2} O_{j}}{\partial x_{j}^{2}}\left(x_{y}^{j}, z\right)=-2 \alpha_{j} \frac{n_{j}}{n m_{j}(x)}<0 .
$$


As a consequence, the sign of $\varphi^{\prime}$ is simply

$$
\operatorname{sign}\left(\varphi^{\prime}(z)\right)=\operatorname{sign}\left(\frac{\partial F_{x}^{j}}{\partial z}(z, \varphi(z))\right) .
$$

Let us consider $z=\pi_{j}$. We have

$$
\frac{\partial F_{x}^{j}}{\partial z}(z, y)=\frac{n_{j} \beta_{j}}{n m_{j}(x)}>0 .
$$

Thus, the function $\pi_{j} \mapsto x_{j}^{\star}\left(\pi_{j}\right)$ is increasing.

Let $z$ be the sensitivity coefficient $\beta_{j}$. We have

$$
\frac{\partial F_{x}^{j}}{\partial z}(z, y)=\frac{n_{j}}{n}\left(-2 \beta_{j} \frac{y}{m_{j}(x)}+1+\frac{\pi_{j}}{m_{j}(x)}\right) .
$$

Using $F_{x}^{j}(z, \varphi(z))=0$, it leads to

$$
\frac{\partial F_{x}^{j}}{\partial z}(z, \varphi(z))=\frac{n_{j}}{n} \frac{-1}{z}<0 .
$$

Thus, the function $\beta_{j} \mapsto x_{j}^{\star}\left(\beta_{j}\right)$ is decreasing. In such a case of an inactive constraint, the premium equilibrium is independent of the initial portfolio size $n_{j}$. i.e.

When the solvency constraint is active, the premium equilibrium $x_{j}^{\star}$ verifies $g_{j}^{1}\left(x_{j}^{\star}\right)=0$,

$$
x_{j}^{\star}=\pi_{j}+\frac{k_{995} \sigma(Y) \sqrt{n_{j}}-K_{j}}{n_{j}\left(1-e_{j}\right)} .
$$

Here, the implicit function theorem is not necessary since $x_{j}^{\star}$ does not depend on $x_{-j}^{\star}$. We deduce that $x_{j}^{\star}$ is an increasing function of $\pi_{j}, k_{995}, \sigma(Y), e_{j}$ and a decreasing function $K_{j}$.

The function $n_{j} \mapsto x_{j}^{\star}\left(n_{j}\right)$ is not necessarily monotone. Let $z$ be $n_{j}$. Differentiating Equation (11) with respect to $z$, we get

$$
\varphi^{\prime}(z)=\frac{1}{z^{3 / 2}\left(1-e_{j}\right)}\left(-\frac{k \sigma(Y)}{2}+\frac{K_{j}}{\sqrt{z}}\right),
$$

whose sign depends on the value of the other parameters.

Proposition 2.3. Let $x^{\star}$ be the premium equilibrium of the insurance game with I insurers. When no constraint functions are active, the premium equilibrium solves a linear system of equations $M_{\beta} x^{\star}=v$, where

$$
M_{\beta}=\left(\begin{array}{cccc}
2 \beta_{1} & -\frac{1+\beta_{1}}{I-1} & \ldots & -\frac{1+\beta_{1}}{I-1} \\
& \ddots & & \\
-\frac{1+\beta_{I}}{I-1} & \cdots & -\frac{1+\beta_{I}}{I-1} & 2 \beta_{I}
\end{array}\right) \quad \text { and } v=\left(\begin{array}{c}
\beta_{1} \pi_{1} \\
\vdots \\
\beta_{I} \pi_{I}
\end{array}\right) .
$$


Proof. As, no constraint functions are active, if $x^{\star}$ is a Nash equilibrium, $x^{\star}$ must verify for all $j$

$$
\nabla_{x_{j}} O_{j}\left(x^{\star}\right)=0 \Leftrightarrow 2 \beta_{j} x^{\star}-\left(1+\beta_{j}\right) \frac{1}{I-1} \sum_{k \neq j} x_{k}^{\star}=\beta_{j} \pi_{j} .
$$

The latter equation can be rewritten as $M_{\beta} x=\beta \circ \pi$. The matrix $M_{\beta}$ is diagonally dominant if and only if for all $j$

$$
\left|2 \beta_{j}\right|>\sum_{k \neq j}\left|-\frac{1+\beta_{j}}{I-1}\right| \Leftrightarrow \beta_{j}>1 .
$$

Having positive diagonal terms, $M_{\beta}$ is invertible, see e.g. Horn \& Johnson (1990). Thus, $x^{\star}=M_{\beta}^{-1} \beta \circ \pi$. In the special case where $\beta_{j}=\beta$ for all $j, M_{\beta}$ is a Toeplitz matrix.

Proposition 2.4. The insurance game with I insurers with objective function (5) and solvency constraint function (7) admits a Stackelberg premium equilibrium.

Proof. By Proposition 2.1, the subgame for followers has a unique Nash equilibrium $x_{-1}^{\star}\left(x_{1}\right)$ for all $x_{1} \in[\underline{x}, \bar{x}]$. By the continuity of objective functions $O_{i}$ and the strict concavity of functions $x_{i} \mapsto O_{i}(x)$, Berge's maximum theorem (see e.g. Aubin (1998)) implies that the function $x_{1} \mapsto x_{-1}^{\star}\left(x_{1}\right)$ is continuous. Therefore, the leader game consists in maximizing on the interval $[\underline{x}, \bar{x}]$ the continuous function $x_{1} \mapsto O_{1}\left(x_{1}, x_{-1}^{\star}\left(x_{1}\right)\right)$. Hence, the function $x_{1} \mapsto O_{1}\left(x_{1}, x_{-1}^{\star}\left(x_{1}\right)\right)$ attains its maximum on the interval $[\underline{x}, \bar{x}]$.

\section{Numerical illustration}

All numerical applications are carried out with the R software, R Core Team (2012), cf. B for computation details. We start by describing the reference parameters in Section 3.1 for which we give the Nash premium equilibrium in Section 3.2. Then, we continue with a sensitivity analysis in Section 3.3 and an analysis of the distribution of the capital at the end of the game in Section 3.4 for the Nash premium equilibrium. Finally, we compare the results with the Stackelberg equilibrium in Section 3.5.

\subsection{Reference parameters}

We consider a game with three insurers operating a 10000 -customer insurance market, i.e. $n=10000, I=3$. Insurer initial portfolio sizes are $\left(n_{1}, n_{2}, n_{3}\right)=(4500,3200,2300)$. The portfolio size is chosen such that Insurer 1 is the leader, Insurer 2 the challenger and Insurer 3 the outsider with $45 \%, 32 \%$ and $23 \%$ market shares, respectively.

We consider the two types of loss models presented in Section 2.2, i.e. (i) PLN loss model such that $\mathbb{E}(Y)=1, \sigma(Y)=4.472$ and (ii) NBLN loss model such that $\mathbb{E}(Y)=1, \sigma(Y)=$ 
10.488. They differ only on the assumption of the frequency distribution (and not on the severity distribution). For these two models, we have a coefficient of variation of the loss distribution of 4.472 and 10.488, respectively (which are reasonable magnitudes for practical purposes).

The weight parameters $\omega_{j}$ used in the computation of the insurer break-even premium are $\omega=(1 / 3,1 / 3,1 / 3)$. We choose the actuarially based premiums $\bar{a}_{j, 0}$ 's and the market premium $\bar{m}_{0}$ given in Table 1 . Table 1 also gives the break-even premium $\pi_{j}$. Since the objective functions coincide between the two loss models, we expect premium equilibria to have similar values for both loss models.

\begin{tabular}{cccc|c|ccc} 
& P1 & P2 & P3 & market & P1 & P2 & P3 \\
\hline PLN/NBLN & 1.10 & 1.15 & 1.05 & 1.10 & 1.10 & 1.1166 & 1.0833 \\
\hline & & $\bar{a}_{j, 0}$ & & $\bar{m}_{0}$ & \multicolumn{3}{|c}{$\pi_{j}$} \\
\end{tabular}

Table 1: Premium parameters: $\bar{a}_{j, 0}, \bar{m}_{0}, \pi_{j}$

Before giving the sensitivity parameters $\beta_{j}$, we present the two lapse models used. For customer behavior, we have two parameters $\mu_{j}, \alpha_{j}$ per insurer given a price-sensitivity function, see Equation 2.

\begin{tabular}{cccc} 
& $\mathrm{P} 1$ & $\mathrm{P} 2$ & $\mathrm{P} 3$ \\
\hline$\tilde{\mu}_{j}$ & -2.890 & -2.508 & -2.209 \\
$\tilde{\alpha}_{j}$ & 9.252 & 7.306 & 6.161 \\
\hline $\bar{\mu}_{j}$ & -12.143 & -9.814 & -8.370 \\
$\bar{\alpha}_{j}$ & 9.252 & 7.306 & 6.161 \\
\hline
\end{tabular}

Table 2: Lapse parameters $\bar{\mu}_{j}, \bar{\alpha}_{j}, \tilde{\mu}_{j}, \tilde{\alpha}_{j}$

In Table 2, we give the lapse parameters. Given $x^{1}=(1,1,1)$ and $x^{1.05}=(1.05,1,1)$, lapse parameters are such that $1-p_{1 \rightarrow 1}\left(x^{1}\right)=10 \%$ and $1-p_{1 \rightarrow 1}\left(x^{1.05}\right)=15 \%$ for Insurer 1 (resp. $14 \%$ and $19 \%$ for Insurer 2 and $18 \%$ and $23 \%$ for Insurer 3). Such values of lapse rates are close to actually observed lapse rates e.g. in continental Europe for private motor lines of business. With these lapse parameters, the expected numbers of lost policies when all insurers propose the same premium are 450.1, 448.0 and 414.0. In Figure 1, total lapse rate functions (i.e. $\left.x_{j} \mapsto 1-p_{j \rightarrow j}(x)\right)$ are plotted on the interval $[1,3]$ for $x_{-j}=(1.5,1.75)$. The left-hand plot shows the total lapse rate function when using price-sensitivity function $\bar{f}_{j}$ and the right-hand plot for $\widetilde{f}_{j}$.

Price sensitivity parameters $\beta_{j}$ of objective functions are fitted using $1-\beta_{j}\left(x_{j} / m_{j}(x)-\right.$ $1) \approx p_{j \rightarrow j}(x)$. With $x^{1.05}$, we get $\beta_{j}=\left(1-p_{j \rightarrow j}(x)\right) / 0.05$ and obtain $\left(\beta_{1}, \beta_{2}, \beta_{3}\right)=(3.0,3.8,4.6)$. The remaining parameters are capital values and the expense rates. Capital values $\left(K_{1}, K_{2}, K_{3}\right)$ are set such that the initial solvency coverage ratio is $133 \%$. Expense rates are $\left(e_{1}, e_{2}, e_{3}\right)=$ $(15 \%, 15 \%, 15 \%)$. 

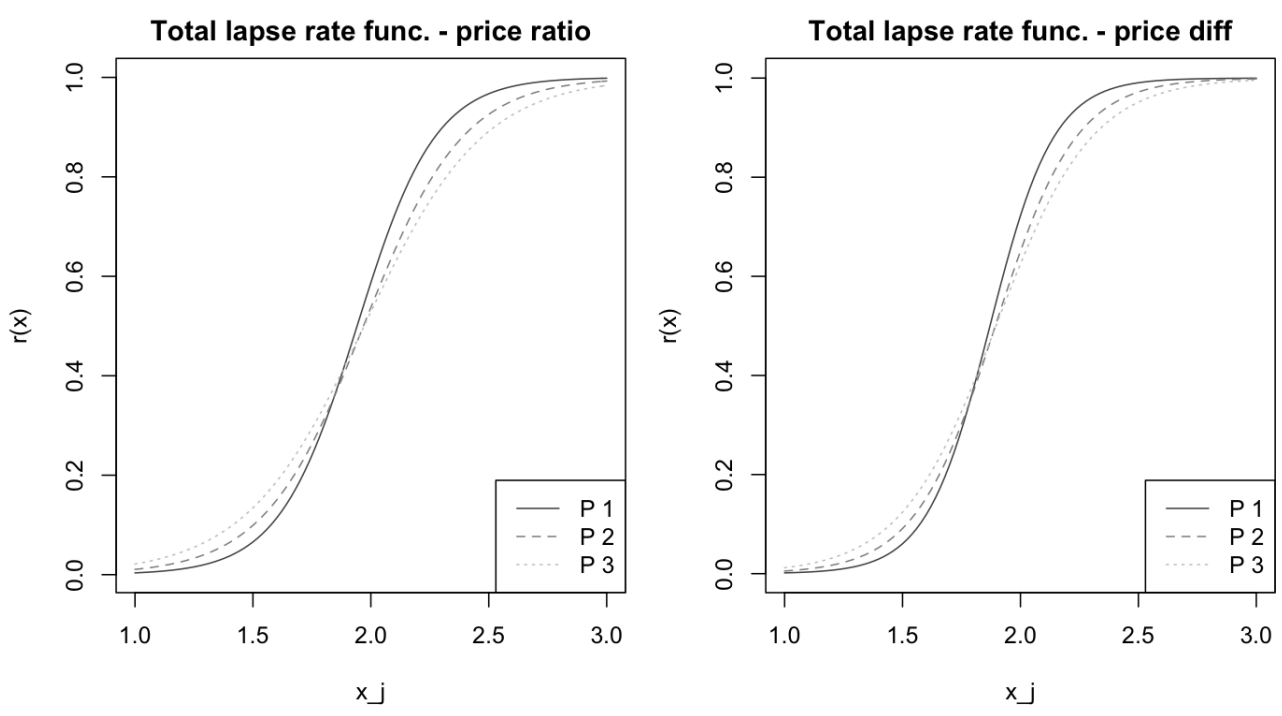

Figure 1: Total lapse rate functions

\subsection{Benchmark results for Nash equilibrium}

Since we consider two loss models (PLN, NBLN) and two price-sensitivity functions $\bar{f}_{j}, \widetilde{f}_{j}$, we define in fact four sets of parameters, which differ on the loss model and the price-sensitivity function. In Table 3 , we report the vector of premium equilibria $x^{\star}$ of the four models in the first three columns. In addition to $x^{\star}$, we also give the expected difference $\Delta_{j}$ in portfolio size (negative means Insurer $j$ expects to lose customers at $x^{\star}$ ) and the estimated solvency coverage ratio $\rho_{j}$ (recalling that the initial solvency coverage ratio is $133 \%$ ) given by

$$
\Delta_{j}=\mathbb{E}\left(N_{j}\left(x^{\star}\right)\right)-n_{j}, \rho_{j}=\frac{K_{j}+\mathbb{E}\left(N_{j}\left(x^{\star}\right)\right)\left(x_{j}^{\star}-\pi_{j}\right)\left(1-e_{j}\right)}{k \sigma(Y) \sqrt{\mathbb{E}\left(N_{j}\left(x^{\star}\right)\right)}} .
$$

\begin{tabular}{l|ccc|ccc|ccc} 
& $x_{1}^{\star}$ & $x_{2}^{\star}$ & $x_{3}^{\star}$ & $\Delta_{1}$ & $\Delta_{2}$ & $\Delta_{3}$ & $\rho_{1}$ & $\rho_{2}$ & $\rho_{3}$ \\
\hline PLN $-\widetilde{f}_{j}$ & 1.544 & 1.511 & 1.471 & -307.1 & -10.58 & 317.7 & 3.524 & 2.993 & 2.729 \\
PLN- $\tilde{f}_{j}$ & 1.544 & 1.511 & 1.471 & -256 & -12.79 & 268.7 & 3.529 & 2.993 & 2.727 \\
NBLN- $\widetilde{f}_{j}$ & 1.544 & 1.511 & 1.471 & -307.1 & -10.58 & 317.7 & 2.295 & 2.042 & 1.881 \\
NBLN- $\tilde{f}_{j}$ & 1.544 & 1.511 & 1.471 & -256 & -12.79 & 268.7 & 2.292 & 2.043 & 1.887 \\
\hline
\end{tabular}

Table 3: Benchmark (Nash) premium equilibrium

The premium equilibrium vector $x^{\star}$ is the same for the four different tested models. We recall that a change in the loss model does not affect the objective function but only the constraint function by changing the standard deviation $\sigma(Y)$ of the aggregate claim distribution. Furthermore, as the parameters of price-sensitivity functions $\bar{f}_{j}, \widetilde{f}_{j}$ are set with the same procedures, the resulting price-sensitivity parameters $\beta_{j}$ are identical. Therefore, having the same break-even parameters $\pi_{j}$, we logically find that the premium equilibrium is the same for the four tested models as long as the constraint functions are not active. 
However, effects of loss models on portfolio sizes and capital levels are strongly different. With $\widetilde{f}_{j}$, any deviation from competitors' premium is more heavily penalized than with $\bar{f}_{j}$. The expected solvency coverage ratio also substantially differs between the two loss models, while the impact of price-sensitivity functions is moderate.

\subsection{Sensitivity analysis to parameters}

In Tables 4 and 5 , we perform a sensitivity analysis considering the NBLN- $\bar{f}_{j}$ model as the reference model. Table 4 reports the analysis with respect to capital (solvency coverage ratios decrease by $60 \%$ ) and price sensitvity parameter (all $\beta_{j}$ increase by $33 \%$ ). Table 5 focuses on actuarially based premiums (all $\bar{a}_{j, 0}$ increase by $33 \%$ ), average market premium $\left(\bar{m}_{0}\right.$ increases by $33 \%$ ) and credibility factors (all $\omega_{j}$ increases by $33 \%$ ). The results of this sensitivity analysis are in line with Proposition 2.2. We observe that any slight difference in one component of premium equilibrium $x^{\star}$ can lead to very different situations of solvency for all insurers. For instance, in Table 4 with the capital down scenario, an increase of the premium equilibrium implies a fall in the expected solvency coverage ratio.

\begin{tabular}{l|ccc|ccc|ccc} 
& $x_{1}^{\star}$ & $x_{2}^{\star}$ & $x_{3}^{\star}$ & $\Delta_{1}$ & $\Delta_{2}$ & $\Delta_{3}$ & $\rho_{1}$ & $\rho_{2}$ & $\rho_{3}$ \\
\hline base & 1.544 & 1.511 & 1.471 & -256 & -12.79 & 268.7 & 2.292 & 2.043 & 1.887 \\
capital down & 1.561 & 1.527 & 1.505 & -212.4 & 22.56 & 189.8 & 1.423 & 1.191 & 1.105 \\
\hline & $x_{1}^{\star}$ & $x_{2}^{\star}$ & $x_{3}^{\star}$ & $\Delta_{1}$ & $\Delta_{2}$ & $\Delta_{3}$ & $\rho_{1}$ & $\rho_{2}$ & $\rho_{3}$ \\
\hline base & 1.544 & 1.511 & 1.471 & -256 & -12.79 & 268.7 & 2.292 & 2.043 & 1.887 \\
price sens. up & 1.406 & 1.385 & 1.353 & -196.7 & -25.26 & 222 & 2.002 & 1.819 & 1.704 \\
\hline
\end{tabular}

Table 4: Sensitivity to capital and price-sensitivity parameters

\begin{tabular}{l|ccc|ccc|ccc} 
& $x_{1}^{\star}$ & $x_{2}^{\star}$ & $x_{3}^{\star}$ & $\Delta_{1}$ & $\Delta_{2}$ & $\Delta_{3}$ & $\rho_{1}$ & $\rho_{2}$ & $\rho_{3}$ \\
\hline base & 1.544 & 1.511 & 1.471 & -256 & -12.79 & 268.7 & 2.292 & 2.043 & 1.887 \\
actuarial up & 1.714 & 1.678 & 1.632 & -256.4 & -20.48 & 276.9 & 2.393 & 2.117 & 1.958 \\
\hline & $x_{1}^{\star}$ & $x_{2}^{\star}$ & $x_{3}^{\star}$ & $\Delta_{1}$ & $\Delta_{2}$ & $\Delta_{3}$ & $\rho_{1}$ & $\rho_{2}$ & $\rho_{3}$ \\
\hline base & 1.544 & 1.511 & 1.471 & -256 & -12.79 & 268.7 & 2.292 & 2.043 & 1.887 \\
market up & 1.884 & 1.841 & 1.796 & -255.6 & -5.798 & 261.4 & 2.494 & 2.202 & 2.021 \\
\hline & $x_{1}^{\star}$ & $x_{2}^{\star}$ & $x_{3}^{\star}$ & $\Delta_{1}$ & $\Delta_{2}$ & $\Delta_{3}$ & $\rho_{1}$ & $\rho_{2}$ & $\rho_{3}$ \\
\hline base & 1.544 & 1.511 & 1.471 & -256 & -12.79 & 268.7 & 2.292 & 2.043 & 1.887 \\
credibility up & 1.544 & 1.513 & 1.469 & -256.7 & -25.59 & 282.3 & 2.292 & 2.038 & 1.89 \\
\hline
\end{tabular}

Table 5: Sensitivity to break-even premium

\subsection{Loss uncertainty analysis}

In Figure 2, we plot the histograms of the capital of Insurer 3 at the end of the game. We consider two of the four models of Table $3: \operatorname{PLN}-\bar{f}_{j}$ and NBLN- $\bar{f}_{j}$ models. In such 
a setting, the premium equilibrium is given by $x^{\star}=(1.544,1.511,1.471)$. In both Figures $2 \mathrm{a}$ and $2 \mathrm{~b}$, the solid vertical line gives the initial capital value $K_{3}$. We observe the huge difference of capital values. The solvency in the NBLN case is much weaker (with $11.4 \%$ of sample capital values below the initial value) than the PLN case (with $0.2 \%$ ). For the PLN case, the premium equilibrium $x^{\star}$ seems rather unfair compared to the actuarial premium. In practice, two scenarios seem natural: (i) some customers may leave the market (because they cannot afford such a high premium) and may start a mutual fund or stay uninsured, (ii) no customer notices this gap for the joy of insurers.

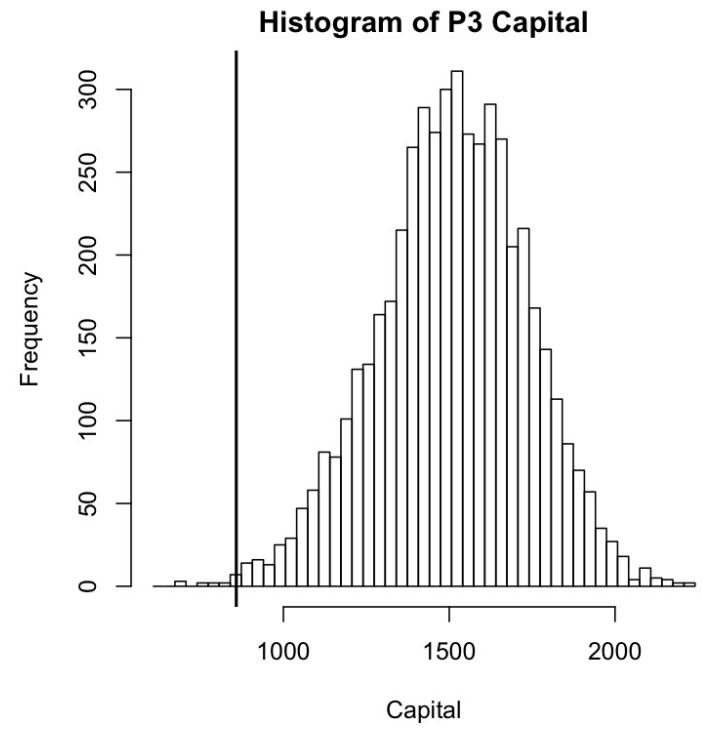

(a) PLN- $\bar{f}_{j}$ model

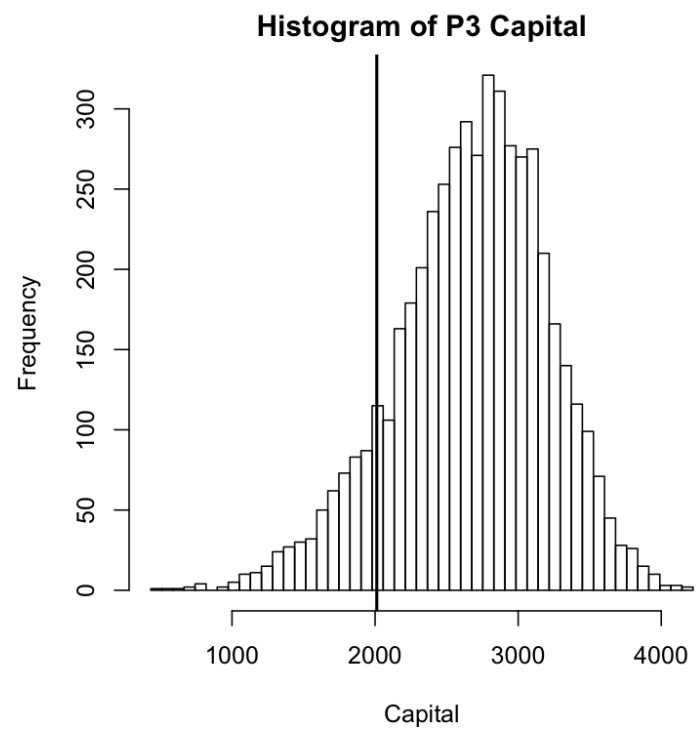

(b) NBLN- $\bar{f}_{j}$ model

Figure 2: Histograms of capital levels (sample size of 5000)

\subsection{Comparison with Stackelberg equilibrium}

Table 6 reports the Stackelberg premium equilibrium. Compared to the Nash equilibrium of Table 3 (where $x^{\star}=(1.544,1.511,1.471)$, the equilibrium value is significantly higher for the Stackelberg equilibrium. We also notice that there is a small difference in terms of the loss model in Table 6, which does not appear in Table 3. In terms of premium ranking, Insurer 1 (the leader) is still higher than competitors, and Insurer 3 asks the cheapest premium at the Stackelberg equilibrium. Regarding the robustness of the premium value, the Stackelberg equilibrium is notably higher than the pure premium $\mathbb{E}(Y)=1$ and also higher than the Nash equilibrium. Finally, the computation complexity is higher for the Stackelberg equilibrium since we carry out a double-level optimization when minimizing the function $x_{1} \mapsto O_{1}\left(x_{1}, x_{-1}^{\star}\left(x_{1}\right)\right)$.

A sensitivity analysis with respect to the initial portofolio size $n_{j}$ has been performed by considering two scenarios: $\left(n_{1}, n_{2}, n_{3}\right)=(3500,3200,3300)$ and $\left(n_{1}, n_{2}, n_{3}\right)=(5500,2700,1800)$. 


\begin{tabular}{l|ccc|ccc|ccc} 
& $x_{1}^{\star}$ & $x_{2}^{\star}$ & $x_{3}^{\star}$ & $\Delta_{1}$ & $\Delta_{2}$ & $\Delta_{3}$ & $\rho_{1}$ & $\rho_{2}$ & $\rho_{3}$ \\
\hline PLN- $\widetilde{f}_{j}$ & 1.740 & 1.598 & 1.554 & -1378 & 389 & 989.1 & 4.265 & 3.408 & 3.128 \\
PLN- $-\tilde{f}_{j}$ & 1.740 & 1.598 & 1.554 & -783.1 & 219.4 & 563.7 & 4.374 & 3.388 & 3.073 \\
NBLN- $\widetilde{f}_{j}$ & 1.740 & 1.598 & 1.554 & -1378 & 389 & 989.1 & 2.737 & 2.175 & 1.973 \\
NBLN- $\tilde{f}_{j}$ & 1.740 & 1.598 & 1.554 & -783.1 & 219.4 & 563.7 & 2.707 & 2.184 & 1.996 \\
\hline
\end{tabular}

Table 6: Stackelberg premium equilibrium

Capital levels $K_{j}$ are determined by using the same coverage ratio $\rho_{i}$. The Stackelberg equilibrium of Table 6 remains unchanged.

\subsection{Sensitivity to the number of insurers}

Our final numerical experiment illustrates the sensitivity of Nash premium and Stackelberg premium with respect to the number $I$ of insurers. For the NBLN loss model and the $\bar{f}_{j}$ sensitivity function, we consider a scenario with 5 insurers by "duplicating" Insurer 2 and Insurer 3. For instance, the initial portfolio sizes are $\left(n_{1}, n_{2}, n_{3}, n_{4}, n_{5}\right)=$ $(4500,3200,3200,2300,2300)$. Results are given in Table 7 . The increase of insurers in the market reduces the value of the equilibrium, both for Nash and Stackelberg equilibria. However, the ranking remains the same as the leader (Insurer 1) still asks the most expensive premium and Insurer 3 the cheapest. For the Stackelberg equilibrium, the leader premium (Insurer 1) is significantly reduced by the addition of two followers (from 1.740 to 1.600), while the reduction is moderate for followers. Note that results for 5 players still hold when the total number of policyholers is renormalize to 10'000 (as in the 3-player game).

\begin{tabular}{rccccc}
$j$ & 1 & 2 & 3 & 4 & 5 \\
\hline (base) Nash eq. & 1.544 & 1.511 & 1.471 & & \\
(base) Stack. eq. & 1.740 & 1.598 & 1.554 & & \\
\hline Nash eq. & 1.531 & 1.494 & 1.494 & 1.450 & 1.450 \\
Stack. eq. & 1.600 & 1.501 & 1.501 & 1.498 & 1.498 \\
\hline
\end{tabular}

Table 7: Comparison of equilibria for 3 and 5 insurers

\section{Conclusion}

This paper assesses the suitability of noncooperative game theory for insurance market modelling. We extend the one-insurer models of Taylor $(1986,1987)$ based on optimal control theory and the subsequent extensions. We also enrich the Bertrand model of Polborn (1998) by refining customer behavior and loss assumptions. Furthermore, the proposed game-theoretic approach contributes to the growing literature of insurance customer behavior modelling, 
e.g. Onn \& Mercer (1998), Dimitriyadis \& Tektas (1998), Yu et al. (2009), Loisel \& Milhaud (2011), Leung \& Kwok (2012), Takagoshi \& Matsubayahi (2013), Chen et al. (2013). The approach proposed in this paper gives a first indicator of the effect of competition on the insurers' solvency. The proposed game models a rational behavior of insurers in setting premiums taking into account other insurers. The ability of an insurer to sell contracts is essential for its survival. In terms of equilibrium concepts, the Nash equilibrium is a natural concept when there is no strong leadership, e.g. in private lines, whereas the Stackelberg equilibrium is relevant for lines manifesting oligopolistic tendencies, typically corporate lines. Numerical illustrations show that the Stackelberg premiums are higher than the Nash premiums and that these premium levels become lower when the number of insurers in the market increases.

The game can be extended in various directions. A natural next step is to consider adverse selection among policyholders, since insurers do not propose the same premium to all customers. A second extension is to model investment results as well as loss reserves and reinsurance treaties. Furthermore, in practice, insurers play an insurance game over several years, gather new information on incurred losses, available capital and competition level. In addition from being dynamic, the market premium shows patterns of cycles with hard and soft phases, known as insurance market cycles, see e.g. Weiss (2007) for a recent survey. Hence, a dynamic game model for insurance markets to explain the occurrence of market cycles could be of particular interest. This will be pursued in a future study.

\section{Acknowledgements}

This work is partially funded by the Research Chair Actuariat Durable sponsored by Milliman, the research chair Management de la modélisation sponsored by BNP Paribas Cardif, the AXA research fund and the Swiss National Science Foundation Project 200021-124635/1. The authors thank the anonymous referees for their useful comments.

\section{References}

Anderson, S. P., Palma, A. D. \& Thisse, J.-F. (1989), 'Demand for differentiated products, discrete choice models, and the characteristics approach', The Review of Economic Studies 56(1), 21-35.

Arrow, K. J. \& Enthoven, A. C. (1961), 'Quasiconcave programming', Econometrica 29(4), 779-800.

Asmussen, S. \& Albrecher, H. (2010), Ruin Probabilities, 2nd edn, World Scientific New Jersey.

Aubin, J.-P. (1998), Optima and Equilibria: An Introduction to Nonlinear Analysis, Springer. 
Bowers, N. L., Gerber, H. U., Hickman, J. C., Jones, D. A. \& Nesbitt, C. J. (1997), Actuarial Mathematics, The Society of Actuaries.

Brockett, P. \& Xia, X. (1995), 'Operations research in insurance: a review', Transactions of Society of Actuaries 47.

Chen, Y., Fang, S.-C. \& Wen, U.-P. (2013), 'Pricing policies for substitutable products in a supply chain with internet and traditionnal channels', European Journal of Operational Research 224(3), 542-551.

Derien, A. (2010), Solvabilité 2: une réelle avancée?, PhD thesis, ISFA.

Dimitriyadis, I. \& Tektas, A. (1998), 'A note on the behavior of the insureds in auto-insurance systems in turkey', European Journal of Operational Research 106(1), 39-44.

Dreyer, V. (2000), Study the profitability of a customer, Master's thesis, Université Louis Pasteur de Strasbourg.

Dutang, C. (2012a), A survey of GNE computation methods: theory and algorithms. Working paper, IRMA.

Dutang, C. (2012b), GNE: computation of Generalized Nash Equilibria. R package version 0.97 .

Emms, P., Haberman, S. \& Savoulli, I. (2007), 'Optimal strategies for pricing general insurance', Insurance: Mathematics and Economics 40(1), 15-34.

Facchinei, F. \& Kanzow, C. (2009), Generalized Nash equilibrium problems. Updated version of the 'quaterly journal of operations research' version.

Fudenberg, D. \& Tirole, J. (1991), Game Theory, The MIT Press.

Geoffard, P. Y., Chiappori, P.-A. \& Durand, F. (1998), 'Moral hazard and the demand for physician services: First lessons from a French natural experiment', European Economic Review 42(3-5), 499-511.

Horn, R. A. \& Johnson, C. R. (1990), Matrix Analysis, Cambridge University Press.

IASB (2008), Non-life insurance contracts, in 'IASB Meeting, Insurance Working Group'.

Kliger, D. \& Levikson, B. (1998), 'Pricing insurance contracts - an economic viewpoint', Insurance: Mathematics and Economics 22(3), 243-249.

Leung, C. \& Kwok, Y. (2012), 'Patent-investment games under asymmetric information', European Journal of Operational Research 223(2), 441-451.

Loisel, S. \& Milhaud, X. (2011), 'From deterministic to stochastic surrender risk models: Impact of correlation crises on economic capital', European Journal of Operational Research $214(2)$.

Malinovskii, V. K. (2010), Competition-originated cycles and insurance companies. work presented at ASTIN 2009. 
McFadden, D. (1981), Econometric Models of Probabilistic Choice, in 'Structural Analysis of Discrete Data with Econometric Applications', The MIT Press, chapter 5.

Mimra, W. \& Wambach, A. (2010), A Game-Theoretic Foundation for the Wilson Equilibrium in Competitive Insurance Markets with Adverse Selection. CESifo Working Paper No. 3412 .

Moreno-Codina, J. \& Gomez-Alvado, F. (2008), 'Price optimisation for profit and growth', Towers Perrin Emphasis 4, 18-21.

Onn, K. P. \& Mercer, A. (1998), 'The direct marketing of insurance', European Journal of Operational Research 109(3), 541-549.

Osborne, M. \& Rubinstein, A. (2006), A Course in Game Theory, Massachusetts Institute of Technology.

Picard, P. (2009), Participating insurance contracts and the Rothschild-Stiglitz equilibrium puzzle. working paper, Ecole Polytechnique.

Polborn, M. K. (1998), 'A model of an oligopoly in an insurance market', The Geneva Paper on Risk and Insurance Theory 23(1), 41-48.

Powers, M. R. \& Shubik, M. (1998), 'On the tradeoff between the law of large numbers and oligopoly in insurance', Insurance: Mathematics and Economics 23(2), 141-156.

Powers, M. R. \& Shubik, M. (2006), 'A "square- root rule" for reinsurance', Cowles Foundation Discussion Paper No. 1521. .

R Core Team (2012), R: A Language and Environment for Statistical Computing, R Foundation for Statistical Computing, Vienna, Austria.

URL: http://www.R-project.org

Rees, R., Gravelle, H. \& Wambach, A. (1999), 'Regulation of insurance markets', The Geneva Paper on Risk and Insurance Theory 24(1), 55-68.

Rosen, J. B. (1965), 'Existence and Uniqueness of Equilibrium Points for Concave N-person Games', Econometrica 33(3), 520-534.

Rothschild, M. \& Stiglitz, J. E. (1976), 'Equilibrium in competitive insurance markets: An essay on the economics of imperfect information', The Quarterly Journal of Economics 90(4), 630-649.

Smith, K., Willis, R. \& Brooks, M. (2000), 'An analysis of customer retention and insurance claim patterns using data mining: a case study', The Journal of the Operational Research Society 51(5), 532-541.

Takagoshi, N. \& Matsubayahi, N. (2013), 'Customization competition between branded firms: continuous extension of product line from core product', European Journal of Operational Research 225(2), 337-352. 
Taylor, G. C. (1986), 'Underwriting strategy in a competitive insurance environment', Insurance: Mathematics and Economics 5(1), 59-77.

Taylor, G. C. (1987), 'Expenses and underwriting strategy in competition', Insurance: Mathematics and Economics 6(4), 275-287.

Teugels, J. \& Sundt, B. (2004), Encyclopedia of Actuarial Science, Vol. 1, John Wiley \& Sons.

Wambach, A. (2000), 'Introducing heterogeneity in the Rothschild-Stiglitz model', Journal of Risk and Insurance 67(4), 579-591.

Weiss, M. A. (2007), 'Underwriting cycles: a synthesis and further directions', Journal of Insurance Issues 30(1), 31-45.

Yu, Y., Huang, G. \& Liang, L. (2009), 'Stackelberg game-theoretic model for optimizing advertising, pricing and inventory policies in vendor managed inventory production supply chains', Computers $\&$ Industrial Engineering 57(1), 368-382.

Zorich, V. (2000), Mathematical Analysis I, Vol. 1, Universitext, Springer.

\section{A Details on the Solvency constraint function}

Currently, European insurers report their solvency margin in the Solvency I framework, based on the maximum of a percentage of gross written premium and aggregate claim mean. According to Derien (2010), a non-life insurer computes its solvency margin as

$$
\mathrm{SM}=\max (18 \% \times \mathrm{GWP}, 26 \% \times \mathrm{AC}) \times \max (50 \%, \mathrm{AC} \text { net of reins } / \mathrm{AC} \text { gross of reins }),
$$

where GWP denotes the gross written premium and $\mathrm{AC}$ the aggregate claim mean*. Discarding reinsurance, the Solvency I framework leads to a solvency margin

$$
\mathrm{SM}=\max (9 \% \times \mathrm{GWP}, 13 \% \times \mathrm{AC}) .
$$

This approach is not really satisfactory, as it does not take into account the risk volality of underwritten business. Since 2005, actuaries are well busy with the upcoming Solvency II framework. In this new framework, the quantitative part leads to the computation of two capital values, both based on the difference between a certain quantile and the mean of the aggregate loss distribution. The solvency capital requirement (SCR) is based on the 99.5\%quantile, whereas the minimum capital requirement (MCR) is based on the 85\%-quantile.

Furthermore, if the solvency requirement is not fulfilled, in Solvency I, the regulator response is immediate: depending on the insolvency severity, regulators can withdraw the

*The percentages $18 \%$ and $26 \%$ are replaced respectively by $16 \%$ and $23 \%$ when the GWP exceeds 57.5 Meur or AC exceeds 40.3 Meur. 
authorisation to underwrite new business or even force the company to go run-off or to sell part of its portfolio. In Solvency II, this happens only when the MCR level is not met. There is a buffer between MCR and SCR where regulators impose some specific actions to help returning to the SCR level.

In our game context, we want to avoid the simplistic Solvency I framework, but still want to keep the tractablity for the SCR computation rule. We recall that the aggregate claim amount is assumed to be a frequency - average severity model. Consider the aggregate claim amount $S_{n}=\sum_{i=1}^{n} Y_{i}$ of $n$ i.i.d. policies and denote by $Q(n, q)$ its $q$-quantile function. We approximate $Q(n, q)$ by a bilinear function of $n$ and $\sqrt{n}$ as follows

$$
Q(n, q)=\mathbb{E}(Y) n+k_{q} \sigma(Y) \sqrt{n},
$$

where the coefficient $k_{q}$ has to be determined and $Y$ is the generic claim amount of a policy. $\mathbb{E}(Y) n$ corresponds to the mean of $S_{n}$, while $k_{q} \sigma(Y) \sqrt{n}$ is related to the standard deviation of $S_{n}$.

Three methods have been tested to compute the solvency coefficient $k_{q}$ : (i) a normal approximation $k_{q}^{N}=\Phi^{-1}(q)$, where $\Phi$ is the distribution function of the standard normal distribution, (ii) a simulation procedure with sample size $10^{5}$ to get $k_{q}^{S}$ as the empirical quantile and (iii) a Panjer recursion to compute the aggregate claim quantile $k_{q}^{P *}$. While the normal approximation is based on the first two moments of the distribution only, simulation and Panjer methods use assumptions on claim frequency and claim severity distributions, see Section 2.2. We also need a policy number $n$. In Table 8, we report solvency coefficients for $n=1000$ policies.

\begin{tabular}{lccccc} 
prob $q$ & $k_{q}^{N}$ & $k_{q}^{P}$-PLN & $k_{q}^{P}$-NBLN & $k_{q}^{S}$-PLN & $k_{q}^{S}$-NBLN \\
\hline 0.75 & 0.674 & 1.251 & 0.913 & 0.649 & 0.627 \\
0.8 & 0.842 & 1.431 & 1.104 & 0.829 & 0.812 \\
0.85 & 1.036 & 1.642 & 1.332 & 1.029 & 1.03 \\
0.9 & 1.282 & 1.912 & 1.627 & 1.299 & 1.312 \\
0.95 & 1.645 & 2.321 & 2.083 & 1.695 & 1.759 \\
0.99 & 2.326 & 3.117 & 2.997 & 2.475 & 2.633 \\
0.995 & 2.576 & 3.419 & 3.352 & 2.777 & 2.976 \\
\hline
\end{tabular}

Table 8: Solvency coefficient $k$

Numerical experiments show that the normal approximation is less conservative for high quantiles (i.e. $k_{q}^{N}<k_{q}^{P}$ ) when the claim number follows a negative binomial distribution, in contrast to the case of the Poisson distribution. Based on this study, we choose to approximate quantiles at the level with coefficient $k_{99.5}=3$.

*See e.g. Theorem 12.4.3 of Bowers et al. (1997). Panjer recursion requires that the claim distribution is discrete. So before using Panjer algorithm, we use a lower discretization of the lognormal claim distribution. 


\section{B Computation details}

The computation of Nash equilibria is based on a Karush-Kuhn-Tucker (KKT) reformulation. We present briefly the problem reformulation and refer the interested readers to e.g. Facchinei \& Kanzow (2009) or Dutang (2012a). In our setting, we have $I$ insurers and three constraints for each of them. For each $j$ of the $I$ subproblems, the necessary KKT conditions are

$$
\begin{array}{r}
\nabla_{x_{j}} O_{j}(x)-\sum_{1 \leq m \leq 3} \lambda_{m}^{j} \nabla_{x_{j}} g_{j}^{m}\left(x_{j}\right)=0, \\
0 \leq \lambda^{j} \perp g_{j}\left(x_{j}\right) \geq 0 .
\end{array}
$$

The inequality part is called the complementarity constraint. A sufficient condition for a point satisfying the KKT conditions to be a generalized Nash equilibrium is that the objective functions are pseudoconcave and a constraint qualification holds. We have seen that objective functions are either strictly concave or pseudoconcave, whereas constraint qualifications are always verified for linear constraints, or strictly monotone functions, see Theorem 2 of Arrow \& Enthoven (1961).

The reformulation proposed uses a complementarity function $\phi(a, b)$ to reformulate the inequality constraints $\lambda^{j}, g_{j}\left(x_{j}\right) \geq 0$ and $\lambda^{j T} g_{j}\left(x_{j}\right)=0$. By definition, a complementarity function is such that $\phi(a, b)=0$ is equivalent to $a, b \geq 0$ and $a b=0$. A typical example is $\phi(a, b)=\min (a, b)$ or $\phi(a, b)=\sqrt{a^{2}+b^{2}}-(a+b)$ called the Fischer-Burmeister function. With this tool, the KKT condition can be rewritten as

$$
\begin{gathered}
\nabla_{x_{j}} L_{j}\left(x, \lambda^{j}\right)=0 \\
\phi .\left(\lambda^{j}, g_{j}\left(x_{j}\right)\right)=0
\end{gathered}
$$

where $L_{j}$ is the Lagrangian function for the subproblem $j$ and $\phi$. denotes the component wise version of $\phi$. So, subproblem $j$ reduces to solving a so-called nonsmooth equation. In this paper, we use the Fischer-Burmeister complementarity function. This method is implemented in the R package GNE of Dutang (2012b). 